\title{
Caracteristicas das Curvas de Regressão da Gonadotrofina Coriônica Pós-mola Hidatiforme Completa
}

\author{
Characteristics of $\beta$-hCG Regression Curves Following Complete Hydatidiform Mole \\ Izildinha Maestá, Marilza V. C. Rudge, José Raimundo S. Passos, Iracema M. P. Calderon \\ Nádia R. Carvalho, Marcos Consonni
}

\section{RESUMO}

Objetivos: construir a curva de regressão do $\beta$-hCG pós-mola hidatiforme completa (MHC) com remissão espontânea e comparar com a curva de regressão pós-MHC com tumor trofoblástico gestacional (TTG). Análise comparativa da curva de regressão do $\beta$-hCG das portadoras de MHC, acompanhadas no Serviço, com a curva de regressão observada por outros autores ${ }^{1-3}$.

Métodos: foi realizada avaliação clínica e laboratorial (dosagem sérica de $\beta$-hCG), na admissão e no segmento pós-molar, de todas as pacientes com MHC, atendidas entre 1990 e 1998 no Hospital das Clinicas de Botucatu - Unesp. O resultado da determinação seriada do $\beta$-hCG foi analisado em curvas log de regressão. A evolução da curva de regressão do $\beta$-hCG foi analisada e comparada em MHC com remissão espontânea e MHC com TTG numa curva log de regressão, com intervalo de confiança de 95\%. A curva log de regressão do grupo de remissão espontânea foi comparada com curvas consideradas padrão ${ }^{1,2}$. Foram construidas curvas log individuais de todas as pacientes e classificadas de acordo com os quatro tipos de curva (I, II, III e IV), propostos para o seguimento pós-molar ${ }^{3}$.

Resultados: 61 pacientes com MHC tiveram seguimento pós-molar completo, 50 (82\%) apresentaram remissão espontânea e 11 (18\%) desenvolveram TTG. No grupo de pacientes com MHC e remissão espontânea, o tempo para alcançar a normalização dos níveis do $\beta$ - $h C G$, após o esvaziamento molar, foi até 20 semanas. As pacientes que desenvolveram TTG apresentaram desvio precoce da curva de regressão normal do $\beta$-hCG, 4 a 6 semanas após o esvaziamento molar. Nestas pacientes, a quimioterapia foi introduzida em média na $9^{a}$ semana pósesvaziamento molar.

Conclusões: a curva de regressão do $\beta$-hCG pós-MHC com remissão espontânea apresentou declinio log exponencial, semelhante ao observado por outros autores ${ }^{1,2}$, e diferente das $M H C$ com TTG. Foram identificados três tipos de curvas de regressão do $\beta$-hCG, semelhantes aos de Goldstein $^{3}$, I, II e IV, e outros dois tipos diferentes de regressão do $\beta$-hCG: V (regressão normal) e VI (regressão anormal).

PALAVRAS-CHAVE: Doença trofoblástica gestacional. Gonadotrofina coriônica. Quimioterapia. Dopplerfluxometria.

\section{Introdução}

A gonadotrofina coriônica humana (hCG) é um hormônio glicoprotéico, de alto peso Departamento de Ginecologia e Obstetrícia da Faculdade de Medicina de Botucatu - Unesp Correspondência: Izildinha Maestá

Departamento de Ginecologia e Obstetrícia Faculdade de Medicina de Botucatu - Unesp Rubião Júnior, $\mathrm{s} / \mathrm{n}^{\circ}$

18618-970 - Botucatu - SP molecular (46.000 dáltons), produzido pelo tecido trofoblástico normal e tumoral. De relevância para a prática clínica, a hCG apresenta propriedades químicas, biológicas e imunológicas ${ }^{4}$.

Tem na sua estrutura química duas cadeias de polipeptídeos, alfa e beta, ligadas por carboidratos. A subunidade alfa é semelhante a dos outros hormônios glicoprotéicos - hormônios luteinizantes $(\mathrm{LH})$, hormônio folículo-estimulante (FSH) e hormônio liberador de tirotropina (TSH). Em contraste, a subunidade beta é que 
determina a atividade biológica e a especificidade imunoquímica da molécula da $\mathrm{hCG}^{4}$. Concentrações de $\beta$-hCG no soro de mulheres saudáveis, não-grávidas, são menores ou iguais a 5 $\mathrm{mUI} / \mathrm{ml}$, que são consideradas o nivel de corte ${ }^{5}$.

O nivel da hCG é o parâmetro mais importante para o acompanhamento de pacientes, após o esvaziamento molar ${ }^{6}$. Marcador biológico do tumor trofoblástico gestacional (TTG), detecta a persistência de trofoblasto ativo nas paredes do útero, ou em outros locais, e fornece dados para a intervenção terapêutica precoce. Nenhum dos métodos de imagem é tão confiável para este propósito como é o marcador tumoral-hCG, conforme salientam três dos maiores grupos de estudo sobre doença trofoblástica gestacional (DTG) ${ }^{6-8}$.

O papel da dosagem de hCG no diagnóstico e no manejo da DTG foi estabelecido por Delfs ${ }^{9}$ e Brewer et al. ${ }^{10}$.

Antes da década de 70 , utilizava-se para a determinação da hCG o teste imunológico semiquantitativo, em urina de 24 horas, pelo método da aglutinação do látex, complementado pelo teste biológico quantitativo do peso do útero do camundongo ${ }^{10}$. O custo e as dificuldades deste método limitavam sua ampla adoção na prática médica.

Em 1972, Vaitukaitis et al. ${ }^{11}$ desenvolveram a técnica de radioimunoensaio (RIE), para dosagem quantitativa da hCG e de sua subunidade $\beta$ no soro ou plasma. Muitos investigadores empregaram este método sensivel e econômico ${ }^{2,12-14}$. Foram desenvolvidos outros métodos sensiveis e específicos, que utilizam anticorpos monoclonais para a determinação da $\beta$-hCG total (molécula intacta de hCG + subunidade $\beta$ livre), no soro ou plasma ${ }^{4}$ : dentre estes, o ELISA, que é um ensaio imunoenzimático, e o MEIA, que é um ensaio imunoenzimático por micropartículas 5 .

O recente sucesso clínico no manejo da DTG está relacionado aos avanços das técnicas de dosagem de hCG, que permitem intervenção terapêutica oportuna ${ }^{6}$. A detecção precoce do TTG, seguida de adequada quimioterapia, promove cura completa, com preservação da capacidade reprodutiva ${ }^{15}$.

O diagnóstico de gravidez molar identifica a paciente que necessita de seguimento clínico e laboratorial ( $\beta$-hCG), e a determinação seriada da gonadotrofina coriônica, no seguimento pósmolar, reconhece as pacientes que necessitam de quimioterapia.

A Sociedade Brasileira de NTG recomenda dosagem de $\beta$-hCG com intervalos semanais, pós-esvaziamento molar, até a obtenção de três dosagens consecutivas normais. Prosseguir com intervalo quinzenal e, a seguir, mensal até completar seis meses após o primeiro resultado negativo. Considera-se doença residual ou persistente, se os níveis séricos de hCG, após o esvaziamento molar, apresentam regressão anormal, com niveis oscilantes e, muitas vezes, crescentes. É também indicativo de doença residual o nivelamento, durante o seguimento pós-molar, da hCG, por três dosagens consecutivas ou seu aumento nesse periodo ${ }^{15,16}$.

A curva de regressão da $\beta$-hCG, pós-esvaziamento molar, identifica o grupo de pacientes com seqüela maligna, isto é, com risco futuro de desenvolver complicações locais (TTG nãometastático) ou metastáticas (TTG metastático), mais do que a mudança histológica da lesão. O diagnóstico precoce permite a cura da maioria das pacientes, com regime quimioterápico de agente único, que é de baixa toxicidade ${ }^{6}$.

A incidência de TTG pós-molar é variável, em decorrência dos diferentes protocolos utilizados para o início da quimioterapia em cada centro de tratamento e da dificuldade para se estabelecer o diagnóstico histopatológico correto de gravidez molar ${ }^{17}$. Na Europa e nos Estados Unidos, a incidência de pacientes tratadas é diferente: cerca de 25\%, nos Estados Unidos, e em torno de $10 \%$, nos centros europeus ${ }^{6}$. No Instituto Nacional de Perinatologia do México ${ }^{18}$, estudo realizado no período de 1988 a 1994 observou incidência de $18 \%$ de TTG pós-molar. No Brasil, vários estudos mostram variação na incidência de TTG pós-molar entre 13 e 31\% ${ }^{19-21}$.

A Organização Mundial da Saúde (OMS) recomenda que o seguimento pós-molar ideal deve ser feito com dosagem seriada de $\beta$-hCG a cada duas semanas, até que os niveis se normalizem, e a cada mês, no primeiro ano. Este trabalho, seguindo as recomendações da $\mathrm{OMS}^{7}$, tem como objetivo construir a curva de regressão da $\beta$-hCG pós-mola hidatiforme completa (MHC) com remissão espontânea e comparar com a curva de regressão pós-MHC com TTG. Outro objetivo é o de verificar as semelhanças ou diferenças da curva de regressão da $\beta$-hCG das portadoras de MHC, acompanhadas no Serviço, com a curva de regressão observada por outros autores $^{1-3}$.

\section{Pacientes e Métodos}

Em 1990, foi criado o Centro de Referência da DTG no Hospital das Clínicas de Botucatu Unesp. Todos os casos foram registrados e o seguimento pós-molar é feito seguindo as recomen- 
dações da $\mathrm{OMS}^{7}$.

Este estudo clínico prospectivo do seguimento pós-molar incluiu pacientes portadoras de MHC que foram diagnosticadas, tratadas e acompanhadas no Hospital das Clínicas de Botucatu - Unesp, no período de 1990 a 1998.

O seguimento pós-molar foi clínico e laboratorial. O seguimento clínico consistiu no estudo das características do ciclo menstrual e no exame ginecológico, analisando a involução do útero e dos cistos do ovário e a orientação para contracepção hormonal, durante um ano. $O$ seguimento laboratorial foi feito pela dosagem sérica de $\beta$-hCG (ELISA/MEIA) pré-esvaziamento molar, e depois, quinzenal até a normalização $(<5 \mathrm{mUI} / \mathrm{mL})$, seguindo-se com dosagens mensais, nos primeiros seis meses, e bimestrais, até completar um ano. A determinação da $\beta$-hCG total (molécula intacta de hCG + subunidade beta livre) foi realizada no Laboratório de Sorologia do Serviço, com o uso do método ELISA, no período de 1990 a 1993, e do método MEIA, a partir de 1994.

Com base na evolução clínico-laboratorial, as pacientes foram classificadas em duas categorias: remissão espontânea ou TTG. Em ambas as condições, foi registrado o número de semanas necessário para a normalização da $\beta$-hCG. Também foi anotado o número de semanas após o esvaziamento molar, para o início da quimioterapia nas pacientes que desenvolveram TTG. Considerava-se a paciente em remissão espontânea após três dosagens consecutivas da $\beta$-hCG $<5 \mathrm{mUI} / \mathrm{mL}$. TTG foi diagnosticado por alteração da curva de regressão do $\beta$-hCG, após o esvaziamento molar, seja pela manutenção dos niveis de $\beta$-hCG (platô) por, pelo menos, três dosagens consecutivas, seja pela elevação (ascensão) de seus títulos.

Após isto, as pacientes foram avaliadas quanto à presença de doença metastática, antes do início da quimioterapia, com os seguintes exames: a) exame ginecológico, com procura de lesão vinhosa nos órgãos genitais externos e na vagina; avaliação do tamanho do útero e da sua consistência e procura de cistos tecaluteínicos; b) ultra-som pélvico com dopplervelocimetria da circulação uterina, para avaliação do volume do útero e dos ovários (cistos tecaluteínicos) e do acometimento miometrial; c) radiografia de tórax, para investigar metástases pulmonares. Esses três exames rastrearam e classificaram os casos com TTG. Depois do tratamento quimioterápico, todas as pacientes foram acompanhadas com dosagens mensais dos níveis da $\beta$-hCG pelo período de dois anos.

O estudo da curva logarítmica exponencial de regressão da $\beta$-hCG pós-MHC foi feito considerando-se a média e os intervalos de confiança de $95 \%{ }^{22}$, em cada momento estudado. Para sua construção, utilizou-se, no eixo $\mathrm{x}$, o tempo zero (pré-esvaziamento) e o tempo em intervalos quinzenais (pós-esvaziamento molar), e, no eixo y, os valores do $\beta$-hCG (mUI/mL), em potências de 10 . A curva log de regressão da $\beta$-hCG das MHC com remissão espontânea foi comparada com a curva de regressão das MHC que tiveram TTG, e com as curvas log de regressão normal observadas por outros autores ${ }^{1,2}$. Foram construidas as curvas log individuais de todas as pacientes com MHC, tanto com remissão espontânea como com evolução para TTG. A seguir, foram analisadas individualmente e classificadas de acordo com os quatro tipos de curva (I, II, III e IV), propostos por Goldstein ${ }^{3}$, para o seguimento pós-molar: o tipo I, caracterizada por queda rápida dos níveis de hCG e ausência de seqüela maligna; o tipo II, padrão de regressão mais lento da hCG e remissão espontânea; o tipo III, com normalização dos títulos de hCG, seguida de ascensão e seqüela maligna em todas as pacientes; o tipo IV, com pequena diminuição inicial dos niveis de hCG, seguida de ascensão e TTG também em todas as pacientes.

O presente trabalho foi aprovado pelo Comitê de Ética em Pesquisa da Faculdade de Medicina de Botucatu - Unesp.

\section{Resultados}

Da série de 61 pacientes com MHC e seguimento pós-molar completo, 50 (82\%) apresentaram remissão espontânea e 11 (18\%) tiveram TTG.

No grupo de pacientes com MHC e remissão espontânea, o tempo necessário para alcançar a normalização dos níveis de $\beta$-hCG, após o esvaziamento molar, foi variável: 43 pacientes $(86 \%)$ até 14 semanas, 47 pacientes $(94 \%)$ até 16 semanas e 50 pacientes $(100 \%)$ até 20 semanas. O número total de amostras utilizadas para dosagem de $\beta$-hCG, neste grupo de pacientes foi de 550, tendo sido feitas, em média, 11 determinações por paciente.

No grupo de pacientes que desenvolveram TTG, o número total de amostras utilizadas para dosagem de $\beta$-hCG foi de 118 , tendo sido feitas 11 determinações por paciente.

Na Figura 1 está a curva de regressão da $\beta$-hCG das pacientes com remissão espontâna e das que desenvolveram TTG. A curva de regressão da $\beta$-hCG das pacientes com MHC e remis- 
são espontânea foi construída considerando-se a média e o intervalo de confiança de $95 \%$ em cada momento estudado. A queda dos níveis de $\beta$-hCG seguiu um declínio log-exponencial.

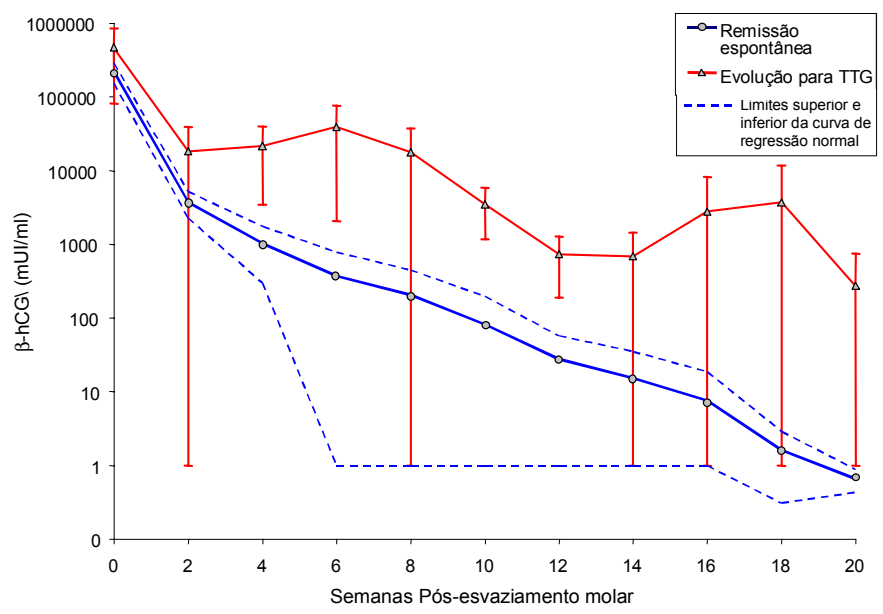

Figura 1 - Curva de regressão da $\beta$-hCG das pacientes com remissão espontânea e das que desenvolveram TTG. Os pontos da curva representam médias e intervalos de confiança a $95 \%$ em cada semana.

A análise comparativa da curva log de regressão normal (remissão espontânea) com a curva de regressão das pacientes que tiveram TTG mostra que, no momento zero (pré-esvaziamento) e na $2^{\text {a }}$ semana pós-molar, não existe diferença entre os grupos com remissão espontânea e com evolução para TTG. Entretanto, nas pacientes com TTG, os valores da $\beta$-hCG aumentaram, acima do limite superior do intervalo de confiança a 95\% da curva normal de regressão, na $4^{\mathrm{a}}$ e $6^{\mathrm{a}}$ semanas seguintes (Figura 1 ).

A quimioterapia foi introduzida em média na 9a semana pós-esvaziamento molar, no grupo de pacientes com TTG. Os valores de $\beta$-hCG, nestas pacientes, continuaram elevados e só são comparáveis ao grupo com remissão espontânea, na $14^{\text {a }}$ semana pós-esvaziamento molar (Figura $1)$.

Do total de 11 pacientes que desenvolveram TTG não-metastático, foi observada curva de regressão de $\beta$-hCG em platô em cinco pacientes, e em ascensão, em seis (Figura 2). Destas 11 pacientes, dez foram tratadas e obtiveram remissão completa da doença e uma paciente recusou tratamento quimioterápico, pois os niveis de $\beta$-hCG estavam em platô prolongado, persistente e baixo $(<1000 \mathrm{mUI} / \mathrm{mL})$. Observa-se, nos casos de TTG com ascensão dos títulos de $\beta$ hCG, início mais precoce da quimioterapia, em torno da $6^{\mathrm{a}}$ a $8^{\mathrm{a}}$ semana pós-esvaziamento molar. Em contraste, houve maior tempo de observação, antes do início do tratamento, nas paci- entes com niveis de $\beta$-hCG em platô baixo (Figura 2).

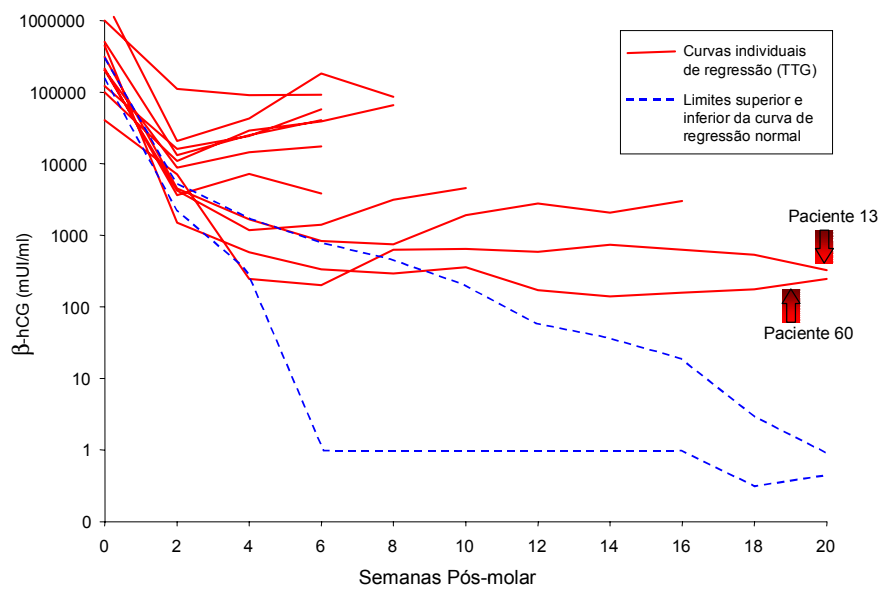

Figura 2-Curvas individuais da $\beta$-hCG de 11 pacientes que desenvolveram TTG, comparadas com a regressão normal (área tracejada) no IC a 95\%. Interrupção nas curvas individuais foi feita no momento do início da quimioterapia, exceto em dois casos, sendo um por recusa ao tratamento (paciente 13) e outro por início tardio da quimioterapia (paciente60).

Nas Figuras 3 e 4 foram analisadas e comparadas as curvas de regressão das $\beta$-hCG das pacientes com remissão espontânea do Hospital das Clínicas de Botucatu, com as de outros autores $^{1,2}$. Observa-se que os intervalos de confiança confluem, em todos os momentos, na curva de Schlaerth et al. $^{2}$ (Figura 3). Só não há superposição na $4^{\text {a }}$ semana pós-esvaziamento molar, na curva de regressão de Morrow et al. ${ }^{1}$ (Figura 4). Portanto, estas três curvas de regressão da $\beta$-hCG podem ser consideradas semelhantes, quando analisadas no intervalo de confiança a $95 \%$.

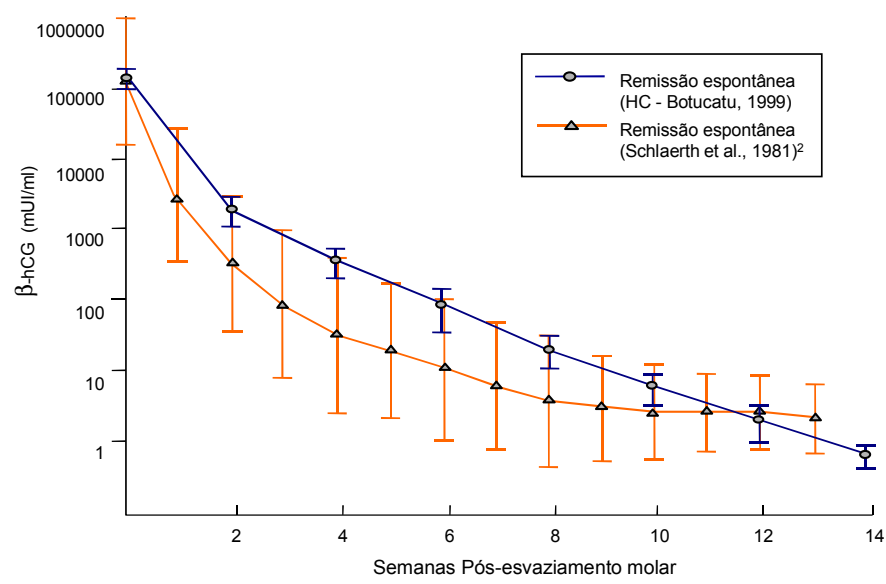

Figura 3 - Comparação das curvas de regressão do $\beta$-hCG das pacientes com remissão espontânea do Hospital das Clínicas de Botucatu com a curva de Schlaerth et al.². 


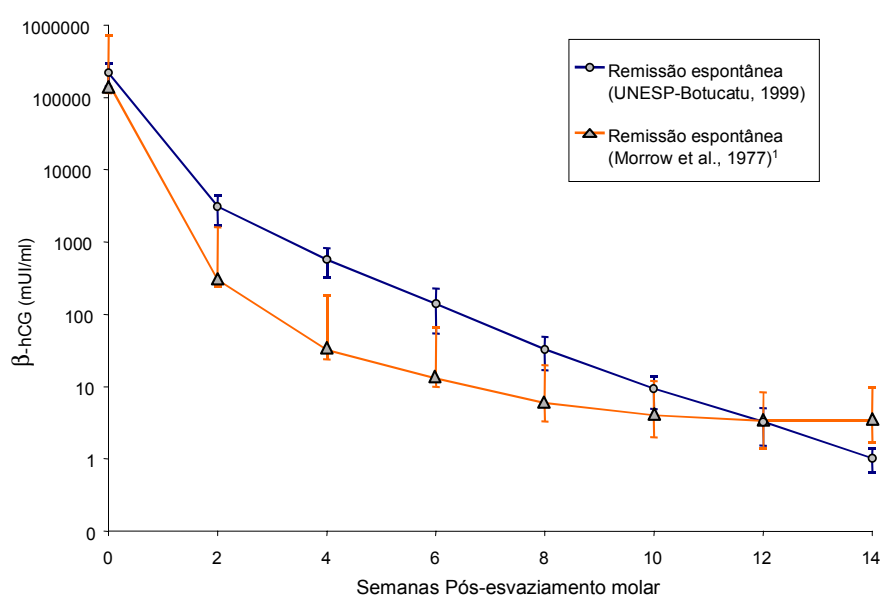

Figura 4 - Comparação das curvas de regressão da $\beta$-hCG das pacientes com remissão espontânea do Hospital das Clínicas de Botucatu com a curva de Morrow et al. ${ }^{1}$.

No Hospital das Clínicas de Botucatu foram identificados vários tipos de curva de $\beta$-hCG, no seguimento pós-molar (Figura 5). Os tipos I e II são semelhantes aos observados por Goldstein ${ }^{3}$ :
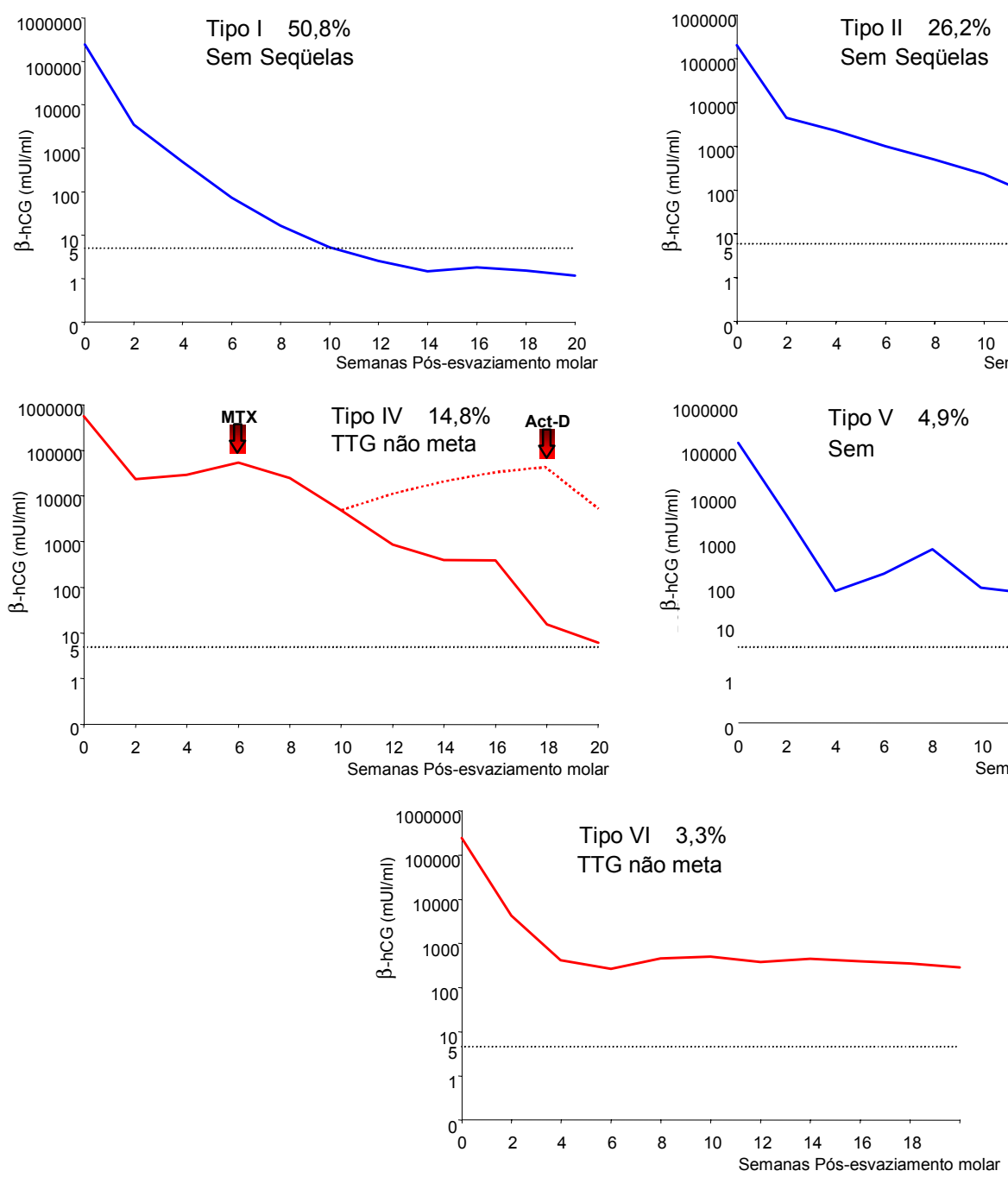

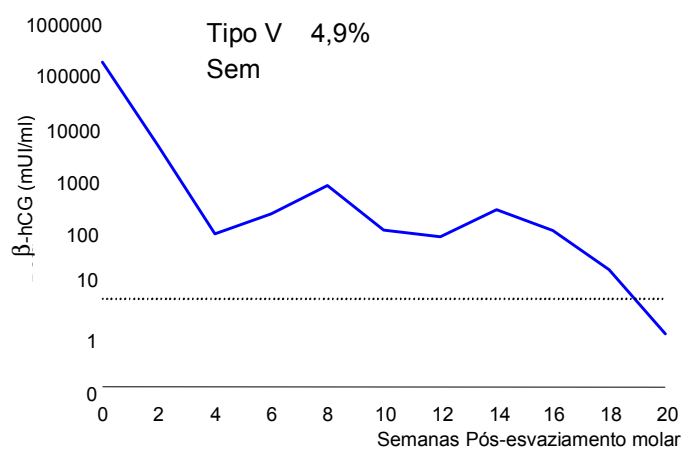

regressão rápida, por volta da $10^{a}$ semana pósesvaziamento molar, no tipo I, e padrão de regressão mais lento, em torno de 16 a 18 semanas pós-esvaziamento molar, no tipo II. Não foi observada nenhuma curva de regressão tipo III. As pacientes que desenvolveram TTG apresentaram, na maioria das vezes, curva de regressão tipo IV semelhante ao de Goldstein: pequena queda inicial, seguida de ascensão dos valores do hCG. Foi verificado o tipo $\mathrm{V}$ da curva de regressão da $\beta$ hCG com nivel oscilatório baixo $(<1000 \mathrm{mUI} / \mathrm{mL}$ e menos de 3 valores alterados) e as pacientes obtiveram remissão espontânea até 20 semanas pós-esvaziamento molar. Outro tipo de curva de regressão da $\beta$-hCG foi encontrado em duas pacientes (tipo VI), caracterizado por diminuição inicial dos niveis de $\beta$-hCG e depois niveis estacionários, formando um platô persistente e baixo $(<1000 \mathrm{mUI} / \mathrm{mL})$ e mais de 3 valores alterados. Uma destas pacientes foi tratada para TTG com 24 semanas pós-esvaziamento molar e a outra recusou tratamento (Figura 2).

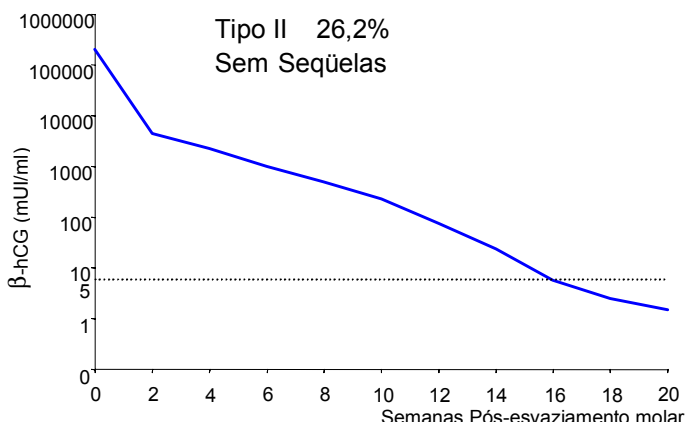

Figura 5-Tipos de curvas de regressão da $\beta$-hCG pós-MHC, identificadas no Hospital das Clínicas de Botucatu: tipo I-regressão rápida, com normalização dos níveis de $\beta$-hCG na $10^{a}$ semana pósesvaziamento molar, observada em $50,8 \%$ das pacientes; tipo II- padrão de regressão lento, com normalização do marcador entre 16 e 18 semanas pós-esvaziamento molar, verificado em $26,2 \%$ das portadoras de MHC; tipo IV - pequena diminuição inicial, seguida de ascensão dos valores de $\beta$-hCG, notada em 14,8\% das pacientes; tipo V - padrão de regressão da $\beta$-hCG oscilatório e baixo, ocorrido em 4,9\% das portadoras de MHC; tipo VI - diminuição inicial dos níveis de $\beta$-hCG, seguida de platô persistente e baixo, observada em 3,3\% dos casos. 
A curva de Goldstein ${ }^{3}$ tipo I foi encontrada em $50,8 \%$ dos casos; a curva tipo II, em $26,2 \%$; a curva tipo III, em $0 \%$; a curva tipo IV, em $14,8 \%$; a tipo V, em $4,9 \%$, e a curva tipo VI, em $3,3 \%$ (Figura 5).

\section{Discussão}

Utilizando a curva log de regressão da $\beta$ hCG no seguimento pós-molar, observamos uma incidência de $18 \%$ de TTG pós-MHC (11 casos). A "Society of Gynecologic Oncologists" cidência de seqüela maligna pós-MHC entre 10 a $25 \%$ dos casos. Entretanto, os autores que utilizaram a curva de regressão da $\beta$-hCG para o diagnóstico de TTG observaram incidência aumentada, variando de 26 a 36\% $\%^{1,2,14}$.

A maioria das pacientes com MHC e remissão espontânea apresentou normalização da curva de regressão da $\beta$-hCG na 14ª semana pósesvaziamento molar, o que também foi observado por outros autores ${ }^{1,2,12,14}$. Todas as pacientes com remissão espontânea obtiveram normalização da $\beta$-hCG até 20 semanas após o esvaziamento molar. Bagshawe et al. ${ }^{23}$ observaram normalização da $\beta$-hCG em 4.066 pacientes $(96,7 \%)$, até 20 semanas após o esvaziamento molar. O intervalo de tempo para a obtenção de níveis normais de $\beta$-hCG pós-MHC com remissão espontânea é variável, e, se a paciente está bem e os títulos de $\beta$-hCG estão caindo, nenhum tratamento adicional é necessário ${ }^{8}$.

Neste estudo, os dados de 50 pacientes com MHC e remissão espontânea permitiram a elaboração de uma curva de regressão de $\beta$-hCG, caracterizada por uma distribuição log normal. Nenhuma dessas pacientes teve aumento subseqüente da $\beta$-hCG e nenhuma recebeu quimioterapia para TTG. Esta observação permite a análise individual dos valores de $\beta$-hCG, no seguimento pós-molar, e serve para orientar o clínico: tranqüilidade, quando os títulos declinam dentro do limite de confiança a 95\%, ou atenção redobrada, se os títulos desviam da curva normal. Portanto, a curva de regressão normal da $\beta$ hCG é útil para o reconhecimento precoce de TTG e de regressão espontânea de MHC e deverá ser utilizada no seguimento pós-molar das pacientes atendidas no Serviço.

A análise comparativa do padrão da curva de regressão da $\beta$-hCG de 11 pacientes que não tiveram queda progressiva até a normalização assinala um grupo separado, que pode ser identificado em torno de 4 a 6 semanas após esvaziamento molar. Neste grupo, as pacientes apre- sentaram títulos de $\beta$-hCG desviando do padrão de regressão normal, mostrando a persistência de atividade trofoblástica e a necessidade de avaliação e tratamento para TTG. Vários autores também identificaram desvio precoce da curva de regressão normal da $\beta$-hCG, em torno de 4 a 6 semanas após o esvaziamento molar, em pacientes que desenvolveram TTG $^{1,2,13}$.

A introdução da quimioterapia, por volta da $9^{a}$ semana pós-esvaziamento molar, tornou os niveis de $\beta$-hCG comparáveis aos do grupo com remissão espontânea, a partir da $14^{a}$ semana de acompanhamento. Rotmensch et al. ${ }^{12}$ compararam a regressão de $\beta$-hCG pós-molar e pós-TTG não-metastático, tratado com quimioterapia. É interessante que obtiveram curvas de regressão semelhantes nesses dois grupos, caracterizadas por um declinio log exponencial.

O diagnóstico de TTG é uniforme entre os autores, quando existem niveis de $\beta$-hCG em ascensão, exame histopatológico de coriocarcinoma, metástases ou hemorragia genital. Entretanto, quando ocorre estabilização dos níveis de $\beta$-hCG (platô) no seguimento pós-molar, não há uniformidade no diagnóstico de TTG nãometastático ${ }^{24}$. Neste estudo, o tempo de observação, antes do início da quimioterapia, quando houve platô na curva de regressão da $\beta$-hCG, variou de 6 a mais de 20 semanas. Múltiplos fatores influenciaram o tempo de espera antes do início da quimioterapia: o nível do platô, presença de hemorragia genital, falha na regressão dos cistos do ovário, antecedente de MHC em gravidez gemelar, segurança de a paciente retornar e resultado do ultra-som transvaginal com Doppler colorido.

Vale ressaltar que a alteração da curva de regressão da $\beta$-hCG foi o fator fundamental no rastreamento e diagnóstico dos TTG. O intervalo de tempo entre a gravidez molar e o diagnóstico de TTG não-metastático foi menor que 6 meses e classificado entre os de baixo risco $^{6-8}$. A taxa de cura nessas pacientes foi de $100 \%$, utilizando-se a quimioterapia (metotrexato e/ou actinomicina D). Apenas uma paciente com prole constituída necessitou de histerectomia em decorrência de foco uterino resistente à quimioterapia.

No acompanhamento pós-molar, recomenda-se a comparação dos níveis de $\beta$-hCG de determinada paciente com uma curva padrão de regressão de pacientes que obtiveram remissão espontânea ${ }^{2,13,14}$. Gal e Friedman, em $1987^{25}$, orientam que a curva de regressão padrão de determinado estudo não pode ser usada de modo universal. Entretanto, quando comparadas as curvas de regressão da $\beta$-hCG das pacientes com 
remissão espontânea do Hospital das Clínicas de Botucatu com as de outros autores ${ }^{1,2}$, não houve diferença significativa. Isto é justificado porque a curva de regressão normal da $\beta$-hCG pós-molar é caracterizada por declínio log exponencial ${ }^{2,12}$, independente da instituição e/ ou população estudadas.

Quando analisadas as curvas log individuais de todas as 61 pacientes, tanto com remissão espontânea como com evolução para TTG, formaram-se grupos de padrão semelhante aos de Goldstein ${ }^{3}$ - curvas tipo I, tipo II e tipo IV - e grupos de padrão diferente - curvas tipo V e tipo VI. A curva tipo I é o protótipo de normalidade e é verificada na maioria das pacientes com remissão espontânea por vários autores ${ }^{1,2,12-14,23}$. A curva tipo II, apesar de ser menos observada pelos autores $^{13,23}$, é considerada de padrão normal e nenhum tratamento adicional é necessário ${ }^{6,8}$. Não observamos a curva tipo III, em nossas pacientes, mas merecem considerações o método utilizado para dosagem da gonadotrofina e a observação de outros autores. O método utilizado por Goldstein ${ }^{3}$ para a determinação da hCG, foi o imunoensaio da fixação do látex, complementado pelo método biológico quantitativo do peso uterino de camundongo. Outros autores que utilizaram a dosagem da $\beta$-hCG quantitativa também não obtiveram padrão de regressão tipo III ${ }^{1,2,14}$. A curva de regressão tipo IV é a mais freqüente entre as pacientes que desenvolveram TTG: pequena queda inicial, seguida de ascensão dos valores da hCG, fato observado também por outros autores ${ }^{2,12-14}$.

O padrão oscilatório dos niveis de $\beta$-hCG, representado na curva tipo $\mathrm{V}$, foi observado em apenas $4,9 \%$ dos casos de MHC. Não se deve consignar pacientes com elevação ocasional (transitória) de $\beta$-hCG como TTG $^{13}$. Escapes transitórios da curva de regressão normal, com subseqüente normalização sem tratamento quimioterápico, foram observados por outros autores ${ }^{2,13,14}$.

Clinicamente importantes, embora de pequeno número, são as pacientes que apresentam títulos em platô tardio, persistente e com niveis baixos, como representado na curva de regressão da $\beta$-hCG tipo VI. Uma das nossas pacientes, com curva tipo VI, manteve-se, no seguimento pós-molar, com platô em torno de 600 $\mathrm{mUI} / \mathrm{mL}$, durante 10 semanas, assintomática, com ultra-som pélvico e radiografia de tórax normais, recusou tratamento e obteve normalização espontânea dos niveis de $\beta$-hCG, na 26aㅗ semana pós-molar. Outra paciente também apresentou platô na curva de regressão, com valores de $\beta$-hCG entre $150-350 \mathrm{mUI} / \mathrm{mL}$, durante 12 se- manas de seguimento pós-molar. Esta paciente mantinha-se assintomática, com ultra-som pélvico e raio-X de tórax normais. Tardiamente, apresentou aumento progressivo dos niveis: de $176 \mathrm{mUI} / \mathrm{mL}$, na $18^{\mathrm{a}}$ semana pós-molar, para $1405 \mathrm{mUI} / \mathrm{mL}$, na 24a semana pós-molar, quando foi iniciada a quimioterapia com pronta remissão.

A presença de platô baixo de $\beta$-hCG, no seguimento pós-molar, é motivo de inquietação no delineamento da conduta médica, porque pode ou não evoluir para TTG ${ }^{24}$. No entanto, desde que a decisão pelo diagnóstico de neoplasia é arbitrário e o final, um evento maligno, consideramos mais razoável, para iniciar o tratamento, um tempo de espera curto do que um tempo de espera longo demais.

A curva de regressão da $\beta$-hCG é o mais sensivel indicador de TTG, e mais estudos com determinação seriada, pós-esvaziamento molar, devem responder questões que ainda inquietam os clínicos que tratam destes raros e tão peculiares tumores.

\section{SUMMARY}

Purpose: to construct a $\beta$-human chorionic gonadotropin $(\beta$ - $h C G)$ regression curve following complete hydatidiform mole (CHM) of patients with spontaneous remission, and then compare it to that of CHM patients with gestational trophoblastic tumor (GTT). Also, to compare the $\beta$-hCG regression curve of CHM patients followed-up at the Service to the regression curve of other authors ${ }^{1-3}$.

Methods: clinical and laboratory evaluations (serum determinations of $\beta-h C G$ ) were performed on admission and during post-molar follow-up of CHM patients treated at the University Hospital of Botucatu between 1990 and 1998. The result of the serial $\beta-h C G$ determinations was analyzed using log regression curves. The evolution of the $\beta$ - $h C G$ regression curve was analyzed and compared between cases of CHM with spontaneous remission and with GTT using a log regression curve, with 95\% confidence interval. The log regression curve of the spontaneous remission group was compared to those of other authors ${ }^{1,2}$. Individual log curves for each patient were constructed and classified according to the four curve types (I, II, III, and IV) proposed by Goldstein ${ }^{3}$ for postmolar follow-up.

Results: sixty-one patients received complete post-molar follow-up, 50 (82\%) showing spontaneous remission and 11 (18\%) developing GTT. In the group of patients with CHM and spontaneous remission, the time to return to normal $\beta$ - $h C G$ levels after mole emptying was 20 weeks. The patients who developed GTT showed early deviation from normal $\beta$ - $h C G$ regression curve 4 to 6 weeks after mole emptying. These patients received chemotherapy normally starting during the 9th post-mole emptying week. 
Conclusions: the regression curve of post-CHM $\beta$ - $h C G$ in patients with spontaneous remission showed a logexponential decline similar to that observed by other authors ${ }^{1,2}$, but different from that of CHM patients who developed GTT. Three types of $\beta$ - $h C G$ regression curves similar to Goldstein' 's , I, II, and IV, were identified, as well as two other different types: $V$ (normal regression) and VI (abnormal regression).

KEY WORDS: Complete hydatidiform mole. $\beta$-hCG regression curve. Gestational trophoblastic tumor. Chorionic gonadotrophin.

\section{Referências}

1. Morrow CP, Kletzky OA, Disaia PJ, Townsend DE, Mishell DR, Nakamura RM. Clinical and laboratory correlates of molar pregnancy and trophoblastic disease. Am J Obstet Gynecol 1977; 128:424-30.

2. Schlaerth JB, Morrow CP, Kletzky OA, Nalick RH, D'Ablaing GA. Prognostic characteristics of serum human chorionic gonadotropin in titer regression following molar pregnancy. Obstet Gynecol 1981; 58:478-82.

3. Goldstein DP. Prophylactic chemotherapy of patients with molar pregnancy. Obstet Gynecol 1971; 38:817-22.

4. Tyrey L. Human chorionic gonadotropin: properties and assay methods. Semin Oncol 1995; 22:121-9.

5. Trundle DS, Chou PP, Raymond A. Automated determination of human choriogonadotropin by use of microparticle capture analysis. Clin Chem 1990; 36:554-6.

6. Society of Gynecologic Oncologists Clinical Practice Guidelines. Practice guidelines: gestational trophoblastic disease. Oncology (Huntingt) 1998; 12:455-61.

7. World Health Organization. Scientific Group on Gestational Trophoblastic Disease. Gestational trophoblastic diseases. Geneva: WHO; 1983. (Technical report series, 692).

8. Management of gestational trophoblastic disease. ACOG Technical bulletin number 178 - March 1993. Int J Gynaecol Obstet 1993; 42:308-15.

9. Delfs E. Chorionic gonadotropin determinations with hydatidiform mole and choriocarcinoma. Ann NY Acad Sci 1959; 80:125-39.

10.Brewer JI, Torok EE, Webster A, Dolkart RE. Hydatidiform mole. A follow-up regimen for the identification of invasive mole and choriocarcinoma and for selection of patients for treatment. Am J Obstet Gynecol 1968; 101:55763.
11.Vaitukaitis JL, Braunstein GD, Ross GT. A radioimmunoassay which specifically measures human chorionic gonadotropin in the presence of human luteinizing hormone. Am J Obstet Gynecol 1972; 113:751-8.

12.Rotmensch J, Rosenshein NB, Block BS. Comparison of human chorionic gonadotropin regression in molar pregnancies and post-molar nonmetastatic gestational trophoblastic neoplasia. Gynecol Oncol 1988; 29:82-6.

13.Yedema KA, Verheijen RH, Kenemans P, et al. Identification of patients with persistent trophoblastic disease by means of a normal human chorionic gonadotropin regression curve. Am J Obstet Gynecol 1993; 168:787-92.

14.Kohorn EI. Hydatidiform mole and gestational trophoblastic disease in Southern Connecticut. Obstet Gynecol 1982; 59:78-84.

15.Abrão FS, Abrão MS, Pereira PP. Neoplasia trofoblástica gestacional não-metastática. In: Madi JM, Grillo BM, editores. Neoplasia Trofoblástica Gestacional. $1^{\mathrm{a}}$ ed. Rio de Janeiro: Revinter; 1995. p.106-11.

16.Belfort $\mathrm{P}$. As sindromes da mola hidatiforme. In: Madi JM, Grillo BM, editores. Neoplasia Trofoblástica Gestacional. 1 ${ }^{\mathrm{a}}$ ed. Rio de Janeiro: Revinter; 1995. p.35-56.

17.Newlands ES, Paradinas FJ, Fisher RA. Recent advances in gestational trophoblastic disease. Hematol Oncol Clin North Am 1999; 13:225-44.

18.Lira Plascencia J, Tenorio Gonzáles F, Gomezpedorso Rea J, et al. Enfermedad trofoblástica gestational: experiencia de 6 años en el Instituto Nacional de Perinatologia. Ginecol Obstet Mex 1995; 63:478-82.

19.Andrade JM, Bighetti S, Kim TYJ, Freitas MMSF. Avaliação de diferentes técnicas de esvaziamento uterino como fator de risco para neoplasia trofoblástica invasora e metastática. AMB Rev Assoc Med Bras 1989; 35:175-8.

20.Yazaki-Sun S, Monteleone PPR, Bertini AM, Camano L. A importância da idade da paciente no prognóstico da mola hidatiforme. Ars Curandi 1992; 25:34-40.

21.Maestá I, Rudge MVC, Michelin OC, et al. Aspectos clínicos, evolutivos e terapêuticos da doença trofoblástica gestacional. J Bras Ginecol 1995; 105:243-8.

22.Bussab WO, Morettin PA. Estatística básica. $4^{\mathrm{a}}$ ed. São Paulo: Atual; 1991.

23.Bagshawe KD, Dent J, Webb J. Hydatidiform mole in England and Wales 1973-83. Lancet 1986; 2:673-7.

24.Kohorn EI. Evaluation of the criteria used to make the diagnosis of nonmetastatic gestational trophoblastic neoplasia. Gynecol Oncol 1993; 48:139-47.

25.Gal D, Friedman M. Follow-up and contraception. In: Szulman AE, Buchsbaum HJ, editors. Gestational Trophoblastic Disease. $1^{\text {st }}$ ed. New York: Springer; 1987. p. 179-85. 